Dyadyura K, Ivakhniuk T, Hrebenyk L, Ivakhniuk Yu, Sukhodub L. Standardization Issues of Test Methods for Engineering Nanomaterials. Abstract book 2nd Grabchenko's international conference on advanced manufacturing processes. InterPartner. Odessa, Ukraine, September 8-11, 2020.

\title{
Standardization Issues of Test Methods for Engineering Nanomaterials
}

\author{
Kostiantyn Dyadyura [ 00000027575971 Tatyana Ivakhniuk [0000 0001-5851-2218 \\ Liudmyla Hrebenyk [0000 0002- 5100763 Uriy Ivakhniuk 0000 0003-2700-5832] \\ Leonid Sukhodub 0000 0002-1559-0475]
}

' Sumy State University, 2, Rymskogo-Korsakova St., Sunny, 40007, Ukraine.

dyadyura@pmtkm.sumdu.edu.ua

\begin{abstract}
The paper relates to the field of standardization of methods for testing by ecotoxicity of engineering nanomaterials using microorganisms. The validity of the methodological approach is discussed in the context of the widespread use of nanomaterials and the high uncertainty of measurement of characteristics to assess their safe application in industry and everyday life. Defining the environmental toxicity of examined samples of $\mathrm{ZnS}$-Alg containing various concentrations of nanoparticles was done in the raw of experiments in vitro according to the level of suppression of growth of test-cultures of Aspergillus niger (1119). Studying the toxic effects of nanoparticles indicated that the medium with ZnS-Alg at concentrations $0.15 \mathrm{gg} / \mathrm{ml}$ and $0.10 \mathrm{pg} / \mathrm{ml}$ had the same average III-d class of toxicity; $0.05 \mathrm{pg} / \mathrm{ml} \mathrm{ZnS}$-Alg had low toxicity. The results of the study have shown that in the standardization of new engineering nanomaterials the nanosafety issue can be addressed by using Aspergillus niger as a test-culture. The obtained data confirm the possibility of using unicellular organisms in the creation of universal express test systems for assessing the safety of nanomaterials. The methodology tested in the work has good prospects and can be included in the protocol in the development of standards, taking into account the ethical aspects of the introduction of modern nanomaterials.
\end{abstract}

Keywords: Standardization, Nanotechnology, Engineered Nanomaterials, Nanosafety, Nanotoxicology, Ecotoxicity

\section{Introduction}

The development of nanotechnologies and nanomaterials is a priority direction for technological innovation, contributing to sustainable and comprehensive economic growth. With the expansion of the production and use of engineering nanomaterials (ENMs) in biomedical, textile, aerospace, cosmetics, petroleum, agricultural and electronic industries, the public's interest is increasingly emphasized in providing risk assessments of the environmental and technological impact of nanotechnological products $[\mathrm{I}]$. This primarily concerns the effects of nano-sized objects, their agglomerates, and aggregates (NOAA) [2,3,4]. The development of standards and infra- 
structure of quality is crucial to maintain the highest quality products and services based on nanotechnology. A responsible approach to the development and commercialization of nanotechnologies requires the standardization of methods that allow assessing the impact of nanomaterials on the environment [5]. Despite a large number of NOAA research findings and the development of new approaches to the complex analysis of the physicochemical parameters of nanoparticles, current toxicological and ecotoxicological methods do not allow to fully evaluate all risks and to determine the potentially dangerous effects of nanomaterials $[1,4,6]$. The lack of a common methodology makes it difficult to systematize the results of different laboratories on the safety of new, diverse nanomaterials. In this regard, there is a need to develop effective methods for testing nanomaterials to identify potential negative impacts on living organisms and ecosystems $[2,5]$. The development of international standards for the correct study of the toxicity and ecotoxicity of NOAA aims to reduce the potential risks of adverse effects, which can significantly simplify the introduction of new technologies and stimulate the development of scientific and technological progress $[7,8]$. Therefore, standardized procedures of ENMs toxicity and ecotoxicity studies need to be further improved and implemented.

Scientific and methodological providing a risk assessment of the impact of innovative nanotechnological products on health and the environment, formation, and progress of technological and regulatory framework is necessary for the development of international standards and recommendations on occupational safety, production, use and disposal of nanomaterials.

\section{Literature Review}

Standardization in the field of nanotechnology provides the following $[9,10,11,12$, 13, 14]:

- detailed study and control of the substance and processes at the nanoscale level (below 100 nanometers in one or more dimensions) for further possible use;

- creation of new improved materials and devices taking into account the properties of nanosized objects, which allow optimizing their practical use.

Existing specific challenges in the development of standards relate to terminology and nomenclature, instrumentation and metrology, testing methods, modeling, and nanoethics standards, including nanosafety [8]. For example, ASTM standards contain terminology in this area, provide recommendations for the practical use of nanotechnology and nanomaterials in various industries, as well as algorithms for properties testing and testing procedures, taking into account health and safety issues.

And furthermore, ISO / IES and CEN standards address nanosafety and nanotoxicology issues. They ensure an assessment of the impact of nanomaterials on living objects during the release of nanoparticles during the life cycle of the device or process. Environmental, health, and safety (EHS) standards require measurement protocols, standard tests, and techniques that can achieve clear comparability of nanomaterial research. In the ISO / TC229 Technical Committee, the Working Group WG 3 (Health, Safety and Environment Scope) is responsible for the development of sci- 
ence-based standards in the field of health, safety and environmental aspects of nanotechnology, the main task of which is to develop standardized methods for:

- the monitoring of professional exposure on nanomaterials;

the identification of relative toxicity and hazard potential of nanomaterials;

- toxicological testing of nanomaterials;

- environmentally sound nanomaterials application;

- making product safety of nanomaterials products.

Nowadays, the regulations to govern the use and implementation of nanomaterials and nanotechnologies have been published. The following standards are used to prepare samples in various appropriate medium for toxicological studies: ISO 10993-18 [9], ISO 14971 [10], ISO/TR 13014 [11], ISO/TR 16197 [12]. ISO/TR 12885:2018

[13]. Information on recommendations for assessment and decision-making for risk management, in the context of incomplete and/or uncertain information on the use of nanomaterials is contained in the following normative instruments [13, 14]. For better harmonization in the field of regulation and control of nanotechnologies, new standardization projects are planned in various industries at the national, regional, and international levels. The actual revision of the ISO / TC 229 documentation allows us to confidently assert the need for a more intensive discussion (Fig. 1) in this direction with more effective use of the results of scientific research of nanoscale objects.

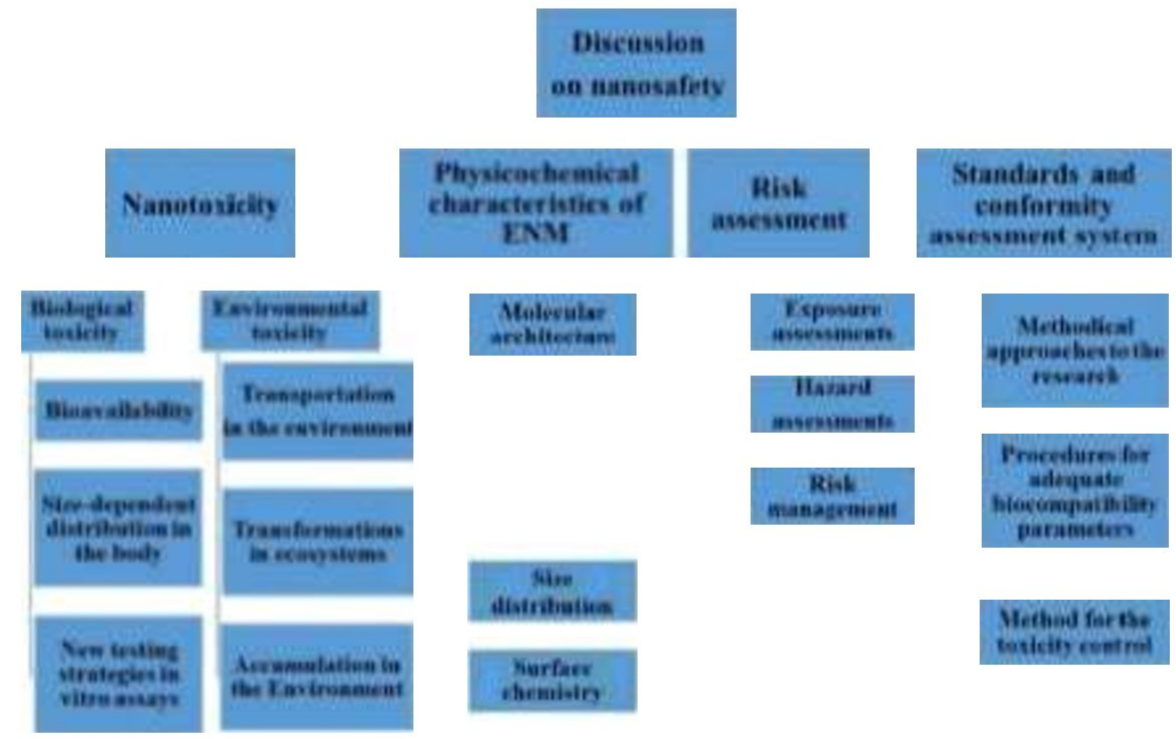

Fig. 1. The discussion scheme of science-based EHS standards for nanotechnologies

When studying nanomaterials, methodological approaches that take into account the behavior of nanoparticles in the process of their interaction with biological objects deserve special attention. Among them are the following four groups:

- epidemiology/occupational medicine; 
- in vivo methods with animals;

- in vitro and in silico methods;

- methods for determining physicochemical properties.

Unfortunately, to date, there is virtually no experimentally proven evidence of the effects of nanoobjects on populations of living organisms as part of an ecosystem. Currently, test-cultures of biological objects are finding to assess the medium toxicity. Strains of bacteria and unicellular fungi, cell cultures of plants and animals actively react to the presence of toxicants in the environment in which they are located.

An analysis of the methods for determining the biosafety of nanomaterials has shown that toxicological screening tests are useful for sound decision-making at the initial stages of research and when developing products with NOAA [2, 5]. In addition, nanoparticle assessment should include toxicokinetic studies that can provide information on potential "target organs" for nanoparticles, their transport and resistance in tissues [7, 8]. In addition, there is the offer to evaluate the dangerous effects of nanomaterials through the study of the possibility of the formation of reactive oxygen species (ROS) in cells, the excess of which provokes the development of oxidative stress with harmful consequences for the whole body. Quantitative estimation of ROS is possible using the electron-spin resonance method [15]. In accordance with current ethical and scientific norms for experimental research, there is a global tendency to replace traditional in vivo studies (using laboratory animals) with improved in vitro model systems (using bacteria, tissue culture, tissue sections) and computer modeling - in silico. Each year, the number of standardized techniques that allow the assessment of the nanotoxicity of nanomaterials using in vitro and in silico methods is increasing. Bio testing is one of the research methods for determination of the degree of the toxic effect of chemical, physical and biological factors of the environment, potentially dangerous for humans and components of ecosystems. The biological test systems that may be used to detect the harmful effects of ENMs include the following test objects: microorganisms; hydrations; organisms of higher animals; cellular and subcellular elements [16].

It is known that for determination of the medium toxicity such cultures as Alternaria alternata, Aspergillus niger, Fusarium moniliforme, Penicillium chrysogenum are successfully used. The reaction of the test-culture to the medium toxicity may be expressed in changes in the quantity and the rate of biomass accumulation, the intensity of excretion or absorption of energy (eg, luminescence for luminescent bacteria), etc. Using test-cultures increasingly displaces a chemical analysis, which involves the detection of chemical toxicants in the environment with the use of specific reagents to the detection of particular substances. Applying biotesting has another advantage. It provides an opportunity to determine the integral effect on living organisms of substances of the environment without determining the chemical composition. The analysis of the literature suggests that the use of unicellular fungi that are common in the environment may be the first step in determining the toxicity of nanoparticles for an ecosystem for further certification of nanoproducts. The aim of this work was studying the possibility of using the test-culture of fungi of the genus Aspergillus, which are involved in the processes of biodegradation and destruction in the environment, for bio testing of synthesized hybrid nanoparticles of zinc sulfide and alginate ( $\mathrm{ZnS}$ $\mathrm{Alg}$ ) with proven antibacterial properties [17]. 


\section{Research Methodology}

Zinc sulfide nanoparticles with alginate $(\mathrm{ZnS}-\mathrm{Alg})$ were synthesized by aqueous chemical solution deposition [17]. As a precursor a mixture of zinc nitrate and thiourea was used (a quantitative ratio was 1: 1). $\mathrm{pH}$ level was raised to 8 by adding an ammonia solution. The optimum temperature of the reactor for chemical synthesis was $90^{\circ} \mathrm{C}$. For making a composite of zinc sulfide with alginate $(\mathrm{ZnS}-\mathrm{Alg}) 3 \%$ sodium alginate solution was supplemented to the mixture. The mineral composition of the synthesized nanomaterial was investigated by X-ray fluorescence analysis (XRF) with using XRF-spectrometer «ElvaX Light SDD» (Ukraine). Morphology of the ZnS-Alg nanoparticles were studied by transmission electron microscopy (TEM). TEM analyses were performed by using "PEM-125k" (Ukraine).

Definition of environmental toxicity, containing various concentrations of examined samples, was done in the raw of experiments in vitro according to the level of suppression of growth of test-cultures of Aspergillus niger (1119) according to patent [12]. Before the inoculating, the quantity of spores in one drop of the prepared suspension was defined according with the help of Horiayev camera. For inoculation, the obtained standard suspension of the Aspergillus niger test culture (1119) was used, which in an amount of $100 \mathrm{mkl}$ was inoculated on Saburo agar medium (LLC "Pharmaktiv", Ukraine) containing various concentrations of the tested substances (Table 1). To control the cultivated of Aspergillus niger (1119) test-culture in isotonic solution $\mathrm{NaCl}$ inoculated in sterile Saburo agar without testing-samples was used.

Table 1. The distribution of test cultures Aspergillus niger (1119) in the experimental series.

\begin{tabular}{|c|c|c|c|c|c|}
\hline 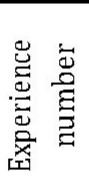 & $\begin{array}{l}\text { In vitrotest design: } \\
\text { A. niger (I119) + } \\
\text { Saburo agar with }\end{array}$ & $\begin{array}{l}\text { The number } \\
\text { of repeat } \\
\text { experiment } \\
\text { (n) }\end{array}$ & 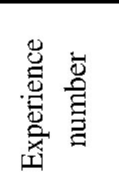 & $\begin{array}{l}\text { In vitrotest design: } \\
\text { A. niger }(1119)+ \\
\text { Saburo agar with }\end{array}$ & $\begin{array}{l}\text { Thenumber } \\
\text { of repeat } \\
\text { experiment } \\
\text { (n) }\end{array}$ \\
\hline 1 & $0.15 \mathrm{pg} / \mathrm{mlZnS}$ & 5 & 6 & $0.05 \mathrm{qg} / \mathrm{ml} \mathrm{Alg}$ & 5 \\
\hline 2 & $0.10 \mathrm{qg} / \mathrm{m} 1 \mathrm{ZnS}$ & 5 & 7 & $0.15 \mathrm{pg} / \mathrm{ml} \mathrm{ZnS-Alg}$ & 5 \\
\hline 3 & $0.05 \mathrm{pg} / \mathrm{m} 1 \mathrm{ZnS}$ & 5 & 8 & $0.10 \mathrm{pg} / \mathrm{ml} \mathrm{ZnS-Alg}$ & 5 \\
\hline 4 & $0.15 \mathrm{pg} / \mathrm{m} 1 \mathrm{Alg}$ & 5 & 9 & $0.05 \mathrm{pg} / \mathrm{ml} \mathrm{ZnS-Alg}$ & 5 \\
\hline 5 & $0.10 \mathrm{pg} / \mathrm{m} 1 \mathrm{Alg}$ & 5 & Control & $\begin{array}{l}\text { A. niger }(1119) * \\
\text { Saburo agar without } \\
\text { testing-samples }\end{array}$ & 5 \\
\hline
\end{tabular}

In the period of incubation on the solid growing medium the diameter of the control and experimentally grown colonies of test-cultures was measured with the help of ruler in tow perpendicular directions and on five surfaces (each 24 hours), and was fixed on the $3,5,7,10$ and 14 day. The number of measurements depended on the speed of growth. The changes in the cultural features of the colonies (nature of edge, change of color and other) were taken into account, start of spore-formation was marked, etc. 
Grounding of the received data the level of suppression of test-culture growth affected by the toxicant in percentage rate to the control group (AR, \%) was defined [17]. Based on the size of index AR the integral estimation of growth for each testculture compared to the control-culture with the further raging of growth range percentage was done: $0 \%-0$ points - no growth; $1-29 \%-1$ point - highly suppressed growth; $30-60 \%-2$ points moderately suppressed growth; $61-90 \%-3$ points weakly suppressed growth; $91 \longrightarrow 100 \%-4$ points - normal growth/stimulation of growth [17]. Depending on the received results of the integral estimation of toxicity (in points) of the examined medium the distribution of the testing samples into the classes of danger was done: I - extremely high toxicity - 0-5 points; II - high toxicity -6 - 29 points; III - moderate toxicity - 30-64 points; IV — weak toxicity - 65-95 points; $\mathrm{V}$ no toxicity $-96-100$ points [17].

\section{Results}

An analysis of the results obtained for synthesized $\mathrm{ZnS}-\mathrm{Alg}$ nanoparticles by using transmission electron microscopy showed that the optimal conditions for chemical synthesis made it possible to obtain particles of the proper spherical shape with the same diameter of spheres. The images obtained at various magnifications made it possible to evaluate the size and structure of nanoparticles (Fig. 2). The average particle size is in the range of from 50 to $80 \mathrm{~nm}$. Each particle has a core formed by $\mathrm{ZnS}$, and a loose shell of the polymer - sodium alginate (Alg). XRF spectrograms of the $\mathrm{ZnS}$-Alg nanocomposite give opportunity to estimate the chemical composition of the nanoparticles and to prove the presence of $\mathrm{ZnS}$ in the test sample. The presence of sodium alginate confirms the presence of a peak in the fluorescence of $\mathrm{Na}$ ions in the "light" spectrum.
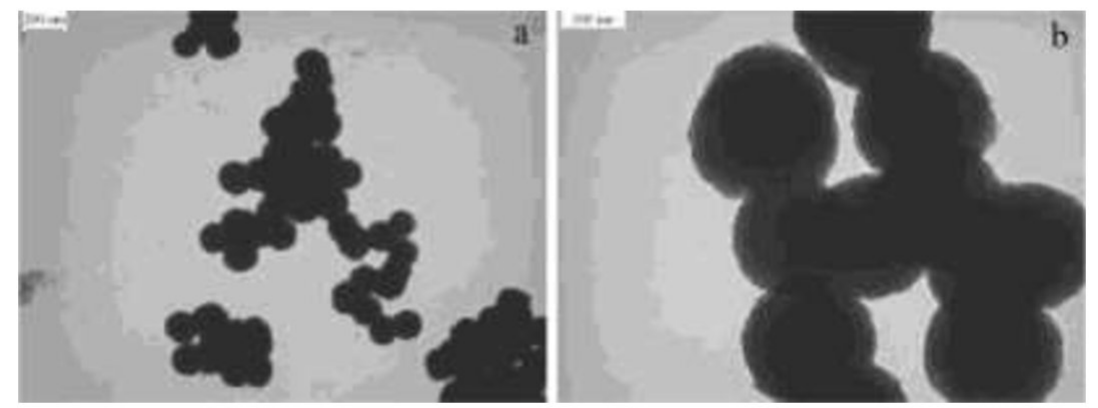

Fig. 2. A TEM images of $\mathrm{ZnS}-\mathrm{Alg}$ nanoparticles

Previous studies of the biological properties of synthesized hybrid $\mathrm{ZnS}$-Alg nanoparticles have demonstrated their ability to inhibit the growth of conditionally pathogenic and pathogenic bacteria [16]. One of the most pressing problems of today is the development of antibiotic resistance in bacterial cultures. That is why the presence of antibacterial properties in the new nanomaterial gives optimistic predictions for its 
further use and dictates the need for studies of the ecotoxicity of these nanoparticles with using test-cultures involved in biodegradation and biodestruction in the environment. Studing the toxicity of $\mathrm{ZnS}$-Alg nanoparticles with using test-cultures of Aspergillus niger (1119) allows to evaluate the degree of exposure to organisms and the ecofunctional characteristics of individual populations of living organisms, their communities and entire ecosystems, and to predict the effects of their use in order to protect environmental effects of anthropogenic pollution and biodegradation. On the basis of the data obtained to the determination of the medium toxicity with different concentrations of the tested compounds ( $\mathrm{ZnS}, \mathrm{Alg}, \mathrm{ZnS}-\mathrm{Alg}$ ), we found that the medium containing $\mathrm{ZnS}$ in concentrations of $0.15 \mathrm{qg} / \mathrm{ml} ; 0.10 \mathrm{qg} / \mathrm{ml} ; 0.05 \mathrm{qg} / \mathrm{ml}$ (experiment 1-3) had a weak toxic effect to the test-culture of Aspergillus niger (1119) with an exposure of 14 days. So, the total indicator AR of the medium with a content of $0.15 \mathrm{pg} / \mathrm{ml} \mathrm{ZnS}$ was $92.4 \%$ (total growth indicator (TGI - 85 points); for $0.10 \mathrm{gg} / \mathrm{ml} \mathrm{ZnS}-93.8 \%$ (TGI - 95 points), for $0.05 \mathrm{pg} / \mathrm{ml} \mathrm{ZnS} \mathrm{-} \mathrm{93.6 \%} \mathrm{(TGI} \mathrm{-} 90$ points), which is typical for class IV of environmental toxicity. Moreover, when evaluating the growth of the test culture colonies on a medium with $0.15 \mathrm{gg} / \mathrm{ml} \mathrm{ZnS}$, a decrease in the toxicity of the medium was revealed during the entire experiment: the culture growth criteria compared to the control on the 3rd - 7th day of the experiment corresponded to 3 points (slightly inhibited growth ), and on the 10th and 14th day - 4 points (normal growth). In determining the toxic effect of alginate (experiment 3-6) against Aspergillus niger (1119) at a 14-day exposure, it was found that media with low alginate concentrations of $0.10 \mathrm{qg} / \mathrm{ml}$ and $0.05 \mathrm{qg} / \mathrm{ml}$ (experiment $4-6$ ) do not have toxicity to the test culture. TGI of the test culture was 100 points ( $\mathrm{V}$ class of toxicity); medium with a content of $0.15 \mathrm{Mtg} / \mathrm{ml} \mathrm{Alg}$ was classified as slightly toxic (AR - 87.6\%; TGI - 80 points), and visually the test-culture had slightly inhibited growth (TGI on 7-10 days was 3 points). Based on comparative data obtained in the study of the toxic effects of $\mathrm{ZnS}$-Alg nanoparticles, it can be concluded that the medium with $\mathrm{ZnS}$ - $\mathrm{Alg}$ at concentrations $0.15 \mathrm{~kg} / \mathrm{ml}$ and $0.10 \mathrm{pg} / \mathrm{ml}$ (experiment 7-8) had the same average III-d toxicity class (TGI was 50 and 60 points respectively); 0.05 $\mathrm{pg} / \mathrm{ml} \mathrm{ZnS}$-Alg (experiment 9) had low toxicity (TGI for a 14-day exposure was 75 points).

\section{Conclusions}

The obtained results suggest that the synthesized hybrid $\mathrm{ZnS}$-Alg nanoparticles with proven antibacterial properties do not have a toxic effect on Aspergillus niger micromycetes, which are involved in biodegradation and biodestruction processes in the environment. Although the biological and environmental impact of the new $\mathrm{ZnS}$-Alg nanoparticles requires further study, the results of the study have shown that in the standardization of new engineering nanomaterials, the nanosafety issue can be addressed by using Aspergillus niger as a test-culture. The research results are promising in the development of technical protocols for studying the environmental toxicity of new nanomaterials but require comparative procedures with already implemented testing methods. Verification of nanomaterials using unicellular 
organisms is express for decision-making on continuing the development of products containing nanomaterial; the expediency of costs for performance of the following stages in need of multilevel testing; availability of appropriate control for the continuation of laboratory studies of nanomaterials. The results of the research can be used to develop international norms on the testing, use, and disposal of nanomaterials for the health and safety of the public, consumers, workers, and the environment using reasonable assumptions and appropriate risk management practices.

\section{References}

1. Gubala, V., Johnston, L. J., Krug, H.F., Moore, C. J., Ober, C. K., Schwenk, M., Vert, M.: Engineered nanomaterials and human health: Part 2. Applications and nanotoxicology (IUPAC Technical Report). Pure Appl. Chem. 90(8) 1325 - 1356 (2018).

2. Jeevanandam, J., Barhoum, A., Chan, Y.S., Dufresne, A., Danquah, M.K.: Review on nanoparticles and nanostructured materials: history, sources, toxicity and regulations. Beilstein J Nanotechnol. 9, 1050 - 1074 (2018).

3. Jahan, S., Yusoff, I.B., Alias, Y.B., Bakar, A.F.B.A.: Reviews of the toxicity behavior of five potential engineered nanomaterials (ENMs) into the aquatic ecosystem. Toxicol Rep. 4, 211220 (2017).

4. Park, H., Yeo, M.: Nanomaterial regulatory policy for human health and environment. Mol. Cell. Toxicol. 12, 223 - 236 (2016).

5. Hodoroaba, V., Ghanem, A.: Nanoscale advances a technique-driven materials categorisation scheme to support regulatory identification. Nanoscale Adv 1, 781791 (2019).

6 Wu, T., Tang, M.: Review of the effects of manufactured nanoparticles on mammalian target organs. J Appl Toxicol 38, 25 - 40 (2018).

7. Kaur, J., Khatri, M., Puri, S.: Toxicological evaluation of metal oxide nanoparticles and mixed exposures at low doses using zebra fish and THP1 cell line. Environ Toxicol 34(4), 375387 (2019).

8. Rauscher, H., Rasmussen, K., Sokull-Kluttgen, B.: Regulatory Aspects of Nanomaterials in the EU. Chem. Ing. Tech. 89, $224-231$ (2017).

9. ISO 10993-18:2020 Biological evaluation of medical devices - Part 18: Chemical characterization of materials.

10. ISO 14971:2019 Medical devices - Application of risk management to medical devices.

11. ISO/TR 13014:2012 Nanotechnologies - Guidance on physico-chemical characterization of engineered nanoscale materials for toxicologic assessmen.

12. ISO/TR 16197:2014 Nanotechnologies - Compilation and description of toxicological screening methods for manufactured nanomaterials.

13. ISO/TR 12885:2018 Nanotechnologies Health and safety practices in occupational settings.

14. ISO/TS 12901-2:2014 Nanotechnologies - Occupational risk management applied to engineered nanomaterials - Part 2: Use of thecontrol banding approach.

15. Fadeel, B., Fornara, A., Toprak, M.S., Bhattacharya, K.: Keeping it real: The importance of material characterization in nanotoxicology. Biochem. Biophys. Res. Commun. 468, $498-503$ (2015).

16. Hrebenyk, L.I., Ivakhniuk, T.V., Sukhodub, L.F.: ZnS quantum dots encapculated with alginate: Synthesis and antibacterial properties. In: Pogrebnjak, A., 
International Conference on Nanomaterials: Applications \& Properties 2017, NAP-2017, pp. 04NB07-1-04NB07-7. IEEE, Piscataway, NJ (2017).

17. Smirnov, V.F.: Method for determining the toxicity of the environment by the degree of inhibition of growth of test cultures of microorganisms. Patent of RU, No. 2570637 (2015). 